\title{
A moist pseudo-incompressible model
}

\author{
W. P. O'Neilla,*, R. Klein ${ }^{\mathrm{a}}$ \\ ${ }^{a}$ Mathematik und Informatik, Freie Universität Berlin, Berlin, Germany
}

\begin{abstract}
A pseudo-incompressible model for moist atmospheric flows is presented. The equation set is derived from a fully compressible system by assuming that the pressure perturbations are small. Unlike the standard dry pseudoincompressible approximation the hydrostatic background state is allowed to vary in time and a set of equations dictating the evolution of the background state are derived. Changes of the background state are the result of net accumulation of diabatic processes and latent heat conversion. Their governing equations emerge in the theory from a consistency condition for the velocity divergence constraint. The model is validated by comparing its results for a well-established benchmark test with those from a fully compressible model. Keywords: Cloud modelling, Sound-proof models, Pseudo-incompressible approximation
\end{abstract}

\section{Introduction}

Atmospheric flows are composed of motions occuring on a large range of temporal and spatial scales. All of these motions can be captured by the compressible Navier-Stokes equations but as sound waves do not play

\footnotetext{
${ }^{*}$ Corresponding author

Email address: warrenon@math.fu-berlin.de (W. P. O'Neill ) 
an important role in these processes, it is theoretically appealing and can be numerically advantageous to remove the sound waves entirely from the governing equations.

Many "sound-proof" equation sets have been developed to tackle this issue. The most well known of these are the Boussinesq equations (Boussinesq (1903)), the anelastic equations (Ogura and Phillips (1962) and Lipps and Helmer (1982)) and the pseudo-incompressible equations (Durran (1989)). These equation sets are derived by introducing different approximations into the governing equations. The Boussinesq approximation replaces the continuity equation by an incompressibility condition, the anelastic approximation disregards the time derivative of the density in the continuity equation to yield a divergence constraint and the pseudo-incompressible approximation ignores the time derivative in the pressure evolution equation which also yields a divergence constraint.

So far only the anelastic equations have been utilised extensively as a basis for modelling moist flows (e.g. Clark (1977), Lipps and Helmer (1982), Grabowski and Smolarkiewicz (2002) and Pauluis (2008)). The Boussinesq equations are unsuitable for moist atmospheric applications due to the assumption of constant density and the pseudo-incompressible equations have yet to be implemented in moist form due to the complications that latent heat causes in the divergence constraint.

In Almgren et al. (2008), a pseudo-incompressible model with source terms and compositional changes for supernovae is developed. Motivated by this work we have created a pseudo-incompressible model for moist atmospheric flows. This model is valid for flows with large density and potential 
temperature variations and is therefore more generally applicable than the anelastic approximation which is only valid for small variations (as shown in Klein (2009)). In the analysis presented in Lipps and Helmer (1982), for example, they require the potential temperature to be a slowly varying function of the vertical coordinate for their moist anelastic model. If we focus on atmospheric motions then this restricts the validity of their model to motions in the troposphere and in this paper they state that "for severe mid-latitude convection...the present analysis is expected to have limited validity". However, the assumption of small density and potential temperature variations is valid for most atmospheric processes of interest and our model is advantageous only in specific cases.

In this paper we will start from the moist compressible model presented in Bryan and Fritsch (2002) and derive our pseudo-incompressible from this equation set. To implement the model numerically we have incorporated it into an in-house finite volume code for low Mach number flow based on the numerics found in Klein (2009). The model is then verified against the moist benchmark test-case given in Bryan and Fritsch (2002).

\section{Derivation of the moist pseudo-incompressible equations}

\subsection{Model assumptions}

We will be making the several simplifications in our model, namely, each phase will have the same temperature and velocity field, we will be ignoring hydrometeor fallout, ice-phase micro-physics, the Coriolis force and sub-gridscale turbulence. 


\subsection{The moist compressible equations}

Making these assumptions we arrive at the following compressible equations with bulk microphysics

$$
\begin{aligned}
& \rho_{t}+\nabla \cdot(\rho \mathbf{u})=0 \\
& (\rho \mathbf{u})_{t}+\nabla \cdot(\rho \mathbf{u} \circ \mathbf{u})+\nabla p=-\rho g \mathbf{k} \\
& \left(\rho q_{v}\right)_{t}+\nabla \cdot\left(\rho q_{v} \mathbf{u}\right)=-\rho C \\
& \left(\rho q_{c}\right)_{t}+\nabla \cdot\left(\rho q_{c} \mathbf{u}\right)=\rho C
\end{aligned}
$$

where the $t$ subscript signifies the partial derivative with respect to time, $\rho$ is the total density and $\mathbf{k}$ is the unit vertical vector. Note, any variables not defined here are defined in Appendix A.

We define the potential temperature and ideal gas equations as

$$
\begin{aligned}
& \theta=T\left(p_{\text {ref }} / p\right)^{\left(R / c_{p}\right)} \\
& p=\rho_{a} R T+\rho_{v} R_{v} T=\left(\frac{1+q_{v} / \epsilon}{1+q}\right) \rho R T
\end{aligned}
$$

where $\epsilon=R / R_{v}$ and the prognostic equation for potential temperature will be given as

$$
(\rho \theta)_{t}+\nabla \cdot(\rho \theta \mathbf{u})=\frac{\rho \theta L_{v}}{c_{p} T} C
$$

which is the same equation used in equation set A in Bryan and Fritsch (2002). ??Does this need to be here??

We wish to define a new variable $P$ such that

$$
P=\left(\frac{1+q_{v} / \epsilon}{1+q}\right) \rho \theta=\frac{p_{\text {ref }}}{R}\left(\frac{p}{p_{\text {ref }}}\right)^{1 / \gamma}
$$


where (5) and (6) have been used to get the second equality and $\gamma=c_{p} / c_{v}$. Now, using (7) and (8) we get the following prognostic equation for $P$

$$
P_{t}+\nabla \cdot(P \mathbf{u})=S
$$

where $S=\left[\frac{L_{v}}{c_{p} T}-1 /\left(\epsilon+q_{v}\right)\right] P C$. Equation (9) taken with equations (1)(4) are the governing equations for the compressible system using our new variable $P$ and are starting point for the derivation of the moist pseudoincompressible equations.

\subsection{The pseudo-incompressible approximation}

Similarly to the pseudo-incompressible derivations presented in Durran (1989) and Almgren et al. (2008) we will start by assuming that the pressure does not vary much from its hydrostatic background value and can be written as

$$
p=p_{0}(z, t)+p^{\prime}(\mathbf{x}, t)
$$

where $p^{\prime} / p_{0}<<1$ and

$$
\frac{\partial p_{0}}{\partial z}=-\rho_{0} g
$$

Note, that unlike in Durran (1989) we have allowed the hydrostatic values to vary in time. This will solve the problem presented by the latent heat term in the divergence constraint by allowing the changing background state to compensate for heating effect and in the idealised dry test-case presented in Almgren (2000) it was shown to play a vital role in ensuring that the solution converges to the compressible solution in the limit of a small heating rate. 
We then set $p=p_{0}$ in (8) to get

$$
P\left(p_{0}, t\right)=P_{0}(z, t)=\frac{p_{\text {ref }}}{R}\left(\frac{p_{0}}{p_{\text {ref }}}\right)^{1 / \gamma} .
$$

Now, we can re-write the evolution equation (7) as the following divergence constraint

$$
\nabla \cdot\left(P_{0} \mathbf{u}\right)=S-\left(P_{0}\right)_{t}
$$

Normally the evolution equation (7) is solved which restricts our time step to that of the acoustic wave time scale but instead we will impose the divergence constraint (13) which filters sound-waves and allows us to take larger timesteps. We must now derive an equation which determines $\left(P_{0}\right)_{t}$.

\subsection{Evolution of the background state}

Using Almgren et al. (2008) we let $\mathbf{u}=\tilde{\mathbf{u}}+w_{0} \mathbf{k}$, where $w_{0}$ is the base-state velocity and $\tilde{\mathbf{u}}$ governs the remaining local dynamics such that $\int_{x_{\min }}^{x_{\max }} \tilde{\mathbf{u}} \cdot \mathbf{k} \mathrm{d} x=$ 0. This means that the domain-wide changes in the vertical flux due to heating are entirely incorporated into $w_{0}$. Using equation (13), the definition for $\tilde{\mathbf{u}}$ and using horizontal solid-wall boundary conditions we can derive the following equation for the background state

$$
\left(P_{0}\right)_{t}+\frac{\partial P_{0} w_{0}}{\partial z}=\bar{S}
$$

where $^{-}=\frac{1}{L} \int_{x_{\min }}^{x_{\max }} \cdot \mathrm{d} x$. Note, $(14)$ is also valid for periodic horizontal boundary conditions.

Using (12) we rewrite equation (14) as

$$
\left(P_{0}\right)_{t}+\frac{\partial P_{0} w_{0}}{\partial z}=\frac{\partial P_{0}}{\partial p_{0}} \frac{\bar{D} p_{0}}{\overline{D t}}+P_{0} \frac{\partial w_{0}}{\partial z}=P_{0}\left(\frac{1}{\gamma p_{0}} \frac{\bar{D} p_{0}}{\overline{D t}}+\frac{\partial w_{0}}{\partial z}\right)=\bar{S}
$$


where the background material derivative is defined as $\frac{\bar{D}}{\overline{D t}}=\frac{\partial}{\partial t}+w_{0} \frac{\partial}{\partial z}$.

Now, in order to calculate $w_{0}$ we need to calculate a value for the material derivative of the background pressure. To do this we first integrate the hydrostatic balance to get

$$
p_{0}^{t o p}(t)-p_{0}(z, t)=\int_{z}^{z_{t o p}} \rho_{0}\left(z^{\prime}, t\right) g \mathrm{~d} z^{\prime}
$$

where $p_{0}^{\text {top }}(t)=p_{0}\left(z_{\text {top }}, t\right)$. We now take the background material derivative of (16) to get

$$
\frac{\bar{D} p_{0}}{\overline{D t}}=\frac{\partial p_{0}^{t o p}}{\partial t}
$$

since the weight of the columns in the background state do not change due to the fact that we are using solid-wall vertical boundary conditions as is required for the benchmark test-case.

It also possible to derive a model which utilises an open-top boundary condition which would be more commmon for meteorlogical applications. This can be achieved following the method presented in Almgren et al. (2008) by assuming the pressure of a parcel does not change, i.e. $\frac{\bar{D} p_{0}}{\overline{D t}}=0$, rather than assuming that the weight of the columns do not change as done above and then using this fact in (15) to derive an equation for $w_{0}$. Also, a suitable buffer layer would have to be implemented to damp spurious motions approaching the upper boundary.

Integrating (15) from the bottom to the top of the domain in the $z$ direction and re-arranging we get

$$
\frac{\partial p_{0}^{t o p}}{\partial t}=\frac{\int_{z_{\min }}^{z_{\max }} \bar{S} / P_{0} \mathrm{~d} z}{\int_{z_{\min }}^{z_{\max }} 1 / \gamma p_{0} \mathrm{~d} z} .
$$


Now that we have a value for $\frac{\partial p_{0}^{t o p}}{\partial t}$ we can calculate $w_{0}$ by integrating (15) from $z_{\min }$ to $z$ to get a value for the background velocity at height $z$

$$
w_{0}=\int_{z_{m i n}}^{z}\left(\frac{\bar{S}}{P_{0}}-\frac{1}{\gamma p_{0}} \frac{\partial p_{0}^{t o p}}{\partial t}\right) \mathrm{d} z^{\prime} .
$$

The background velocity can then be used to determine $\left(P_{0}\right)_{t}$ using (14).

\subsection{The moist pseudo-incompressible equations}

We now have all the equations we require for a moist pseudo-incompressible model. To make the final step in our derivation we must replace the full density in equations (1)-(4). The pseudo-density is defined the same way as in the original pseudo-incompressible derivation presented in Durran (1989) which is the density calculated at the background pressure but using the full potential temperature, i.e.

$$
\rho^{*}=\rho\left(p_{0}, \theta\right)
$$

Making these adjustments results in our new set of governing equations

$$
\begin{aligned}
& \rho_{t}^{*}+\nabla \cdot\left(\rho^{*} \mathbf{u}\right)=0 \\
& \left.\left(\rho^{*} \mathbf{u}\right)_{t}+\nabla \cdot\left(\rho^{*} \mathbf{u} \circ \mathbf{u}\right)\right)+\nabla p=-\rho^{*} g \mathbf{k} \\
& \left(\rho^{*} q_{v}\right)_{t}+\nabla \cdot\left(\rho^{*} q_{v} \mathbf{u}\right)=-\rho^{*} C \\
& \left(\rho^{*} q_{c}\right)_{t}+\nabla \cdot\left(\rho^{*} q_{c} \mathbf{u}\right)=\rho^{*} C
\end{aligned}
$$

with the following equations for the background state

$$
\frac{\partial p_{0}^{t o p}}{\partial t}=\frac{\int_{z_{\min }}^{z_{\max }} \bar{S} / P_{0} \mathrm{~d} z}{\int_{z_{\min }}^{z_{\max }} 1 / \gamma p_{0} \mathrm{~d} z}
$$




$$
\begin{aligned}
& w_{0}(z, t)=\int_{z_{\min }}^{z}\left(\frac{\bar{S}}{P_{0}}-\frac{1}{\gamma p_{0}} \frac{\partial p_{0}^{t o p}}{\partial t}\right) \mathrm{d} z^{\prime} \\
& \frac{\partial P_{0}}{\partial t}+\frac{\partial P_{0} w_{0}}{\partial z}=\bar{S}
\end{aligned}
$$

and the following equation of state and divergence constraint

$$
\begin{aligned}
& P_{0}=\left(\frac{1+q_{v} / \epsilon}{1+q}\right) \rho^{*} \theta=\frac{p_{\text {ref }}}{R}\left(\frac{p_{0}}{p_{\text {ref }}}\right)^{1 / \gamma} \\
& \nabla \cdot\left(P_{0} \mathbf{u}\right)=S-\left(P_{0}\right)_{t} .
\end{aligned}
$$

Note that $p_{0}$ will be used and not the full pressure when calculating the condensation rate numerically as shown in section Appendix B.3.

It is also possible to write an evolution equation for the background density as

$$
\left(\rho_{0}\right)_{t}+\frac{\partial}{\partial z}\left(\rho_{0} w_{0}\right)=0
$$

Here we have taken advantage of the fact that the density remains symmetric in the horizontal direction for the benchmark test-case but for cases where it is non-symmetric this equation would have an extra term of the form $\frac{\partial}{\partial z}\left(\overline{\rho^{\prime} \tilde{w}}\right)$. Note, equation $(30)$ is presented only for completeness and is not utilised in the model.

The replacement of the evolution equation (9) with the divergence constraint (29) and the fact that $\rho$ and $C$ are calculated using the background pressure are the main differences between the moist pseudo-incompressible equations and the compressible system with which we started.

Comparing to an anelastic equation set we see that the differences are seen in the density. In the anelastic equations the density is set to the initial 
hydrostatic values whereas here the less restrictive "pseudo-density" is used. Also, the divergence constraint in the anelastic approximation is derived from the continuity equation and not the potential temperature equation.

Note, for the remainder of the paper the asterisk will be dropped from the pseudo-density in order to simplify the notation.

\section{Numerics of the moist pseudo-incompressible model}

\subsection{Overview of the numerics}

Equations (21)-(29) were solved using a predictor-corrector finite volume code based on numerical techniques described in Klein (2009). The primary variables, $\rho, \rho \mathbf{u}, \rho q_{v}, \rho q_{c}$, and $P_{0}$ are stored at cell centers, whereas the perturbation pressure $p$ is stored at grid nodes. Fluxes of conserved variables arise at grid cell interfaces as usual in a cell-centered finite volume code.

In a predictor step the divergence constraint is ignored and $P$ is advected in the same way as other variables. Also, the old time level pressure is used in the momentum equation. This results in a reduced order of accuracy in the momentum equation due to the pressure term and also due to advective fluxes that do not yet exactly satisfy the divergence constraint.

These inaccuracies are corrected in the two corrector steps. The advective fluxes are corrected in the first step and the error in the momentum equation due to the usage of the old time level pressure is corrected in the second step.

To calculate the microphysical source terms we use the method of saturation adjustment outlined in Grabowski and Smolarkiewicz (1990) after the predictor step but before the corrector steps. Unlike Grabowski and Smolarkiewicz (1990), we will be using the time-varying hydrostatic pressure 
and not the initial hydrostatic pressure in the source term calculation to get values for the temperature and the vapour saturation mixing ratio. For the test-case presented in Bryan and Fritsch (2002) the difference between using the initial and time-varying pressure is negligable, however, the retention of hydrostatic variations can become important for larger-scale flows as shown in Kurowski et al. (2013).

A more detailed description of the numerics is given in Appendix B.

\section{Comparison with the benchmark test-case}

\subsection{Overview of the benchmark test-case}

To test the accuracy of the model we utilised the benchmark simulation proposed in Bryan and Fritsch (2002). This test-case consists of a saturated and neutrally stratified hydrostatic base-state atmosphere with solid-wall boundary conditions, an initially constant value for the total water mixing ratio of 0.02 and a constant base value for the wet equivalent potential temperature $\theta_{e}$ of $320 \mathrm{~K}$ with a perturbation of warm air placed near the bottom of the domain. The definition for $\theta_{e}$ is given as

$$
\theta_{e}=T\left(\frac{p_{d}}{p_{r e f}}\right)^{-R /\left(c_{p}+c_{p c} q\right)} \exp \left(\frac{L_{v} q_{v}}{\left(c_{p}+c_{p c} q\right) T}\right)
$$

which is taken from Emanuel (1994) and the perturbation $\theta^{\prime}$ is given as

$$
\theta^{\prime}=2 \cos ^{2}\left(\frac{\pi R}{2}\right)
$$

where

$$
R=\sqrt{\left(\frac{x}{x_{r}}\right)^{2}+\left(\frac{z-z_{0}}{z_{r}}\right)^{2}}
$$


$z_{0}=2.0 \mathrm{~km}$ and $x_{r}=z_{r}=2.0 \mathrm{~km}$. Using equations (11), (28) and (31) we can calculate the vertical profiles of $\rho, p, \theta, q_{v}$ and $q_{c}$ given our presribed values for $q$ and $\theta_{e}$.

\subsection{Results}

In the original paper four different compressible equation sets are used. To make a comparison we will look at the one which most closely resembles our own model assumptions which is the one labeled set A. This set uses the same governing equations and evolution equation for potential temperature as were used to derive our pseudo-incompressible equations and are given by equations (5) and (7).

In the original paper the plots of $\theta_{e}$ and the vertical velocity are used to test model validity and here we will do the same. Below the plots produced from our model can be seen in figures (1) and (2). These figures were produced using a constant time-step size of $1.66 \mathrm{~s}$ for $\mathrm{CFL}=0.5$ and using the same $100 \mathrm{~m}$ grid spacing as used in the original paper. 


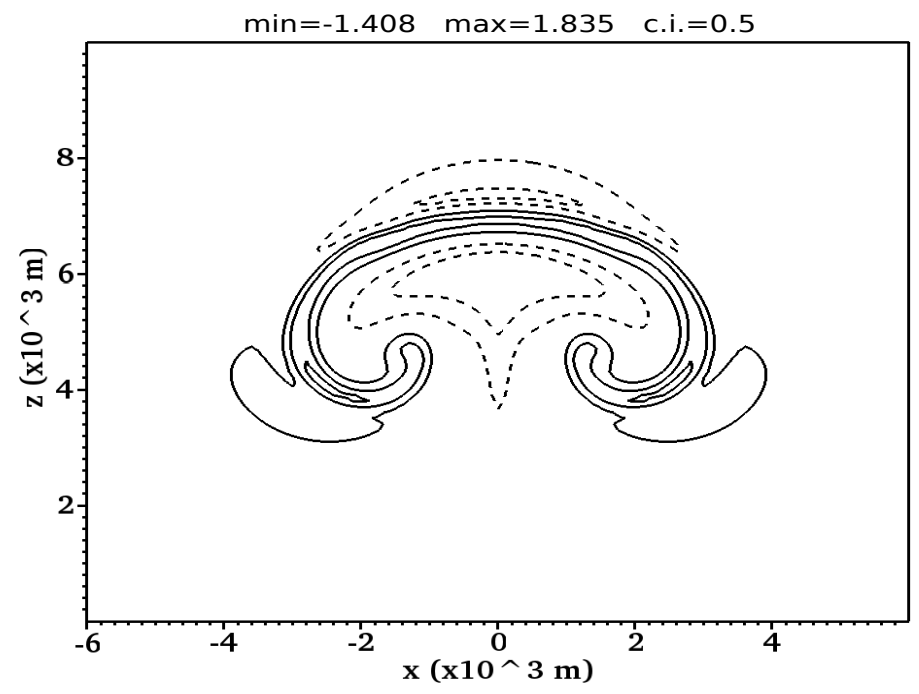

Figure 1: A contour plot of perturbations of the wet equivalent potential temperature at $t=1000 \mathrm{~s}$ for the moist bubble simulation where the contour interval is $0.5 \mathrm{~K}$, the grid spacing is $100 \mathrm{~m}$ and the zero contour is omitted.

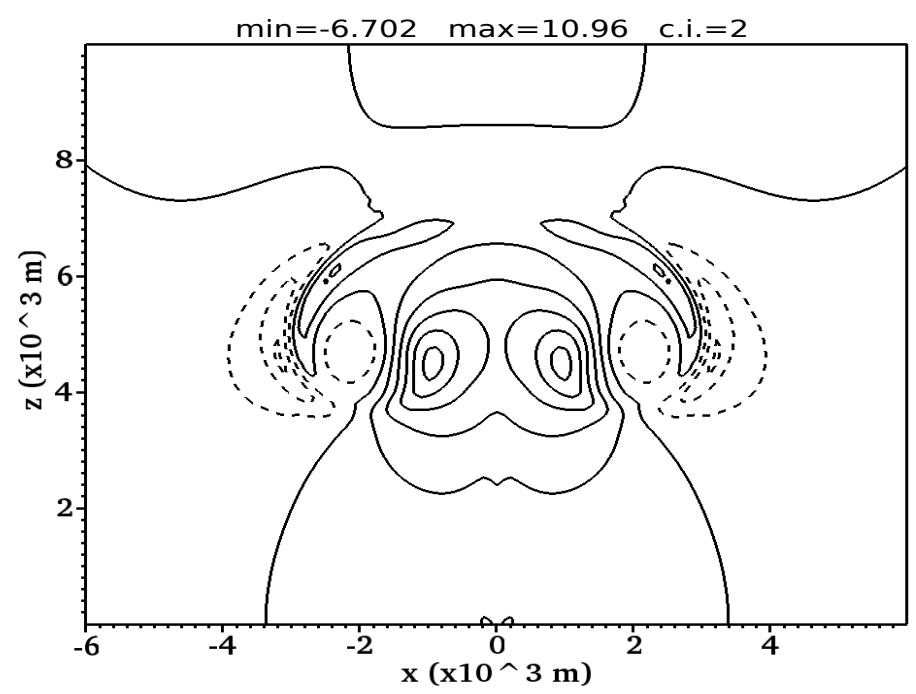

Figure 2: A contour plot of the vertical velocity at $t=1000 \mathrm{~s}$ for the moist bubble simulation where the contour interval is $2 \mathrm{~m} \mathrm{~s}^{-1}$ and the grid spacing is $100 \mathrm{~m}$.

These results compare well qualitatively with those in the original paper 
Bryan and Fritsch (2002), but there are some differences: Our bubble top rises to about $7.1 \mathrm{~km}$ which is slightly higher than the height reached in the original paper of about $6.9 \mathrm{~km}$ giving a difference in the distance covered by the bubbles of about \%4. Looking at the maximum and minimum values we can see that between the vertical velocities there is about a $8 \%$ variation compared to the values in the original paper $\left(10.96 \mathrm{~m} \mathrm{~s}^{-1}\right.$ and $-6.7 \mathrm{~m} \mathrm{~s}^{-1}$ in our case and $11.88 \mathrm{~m} \mathrm{~s}^{-1}$ and $-7.23 \mathrm{~m} \mathrm{~s}^{-1}$ in Bryan's case) and between the wet equivalent potential temperature perturbations there is a variation of about $13 \%$ in the case of the maximum values and a negligible variation in the case of the minimum values $(1.84 \mathrm{~K}$ and $-1.4 \mathrm{~K}$ in our case and $2.14 \mathrm{~K},-1.4 \mathrm{~K}$ in Bryan's case).

There are many differences in our numerics which could account for the discrepancies. For example, our equations are solved in conservation form unlike those in the original paper, our codes use different functions to caculate the condensation rate numerically and they have advection schemes.

In Straka et al. (1993) various models were shown to have large variations in performance when the grid resolution is changed. This illustrated that the numerics have a large role to play in model output. In our case, when we ran the model with a $50 \%$ higher number of cells in the horizontal and vertical directions (i.e. a grid spacing of $66.67 \mathrm{~m}$ ) we found that the maximum and minimum values had much better agreement and some of the qualitative differences in the vertical velocity plot were corrected as shown in figures (3) and (4). In light of this, we can see that even small adjustments to the numerics can influence the overall result and our plots and the one's contained in Bryan and Fritsch (2002) compare well considering their many 
numerical differences.

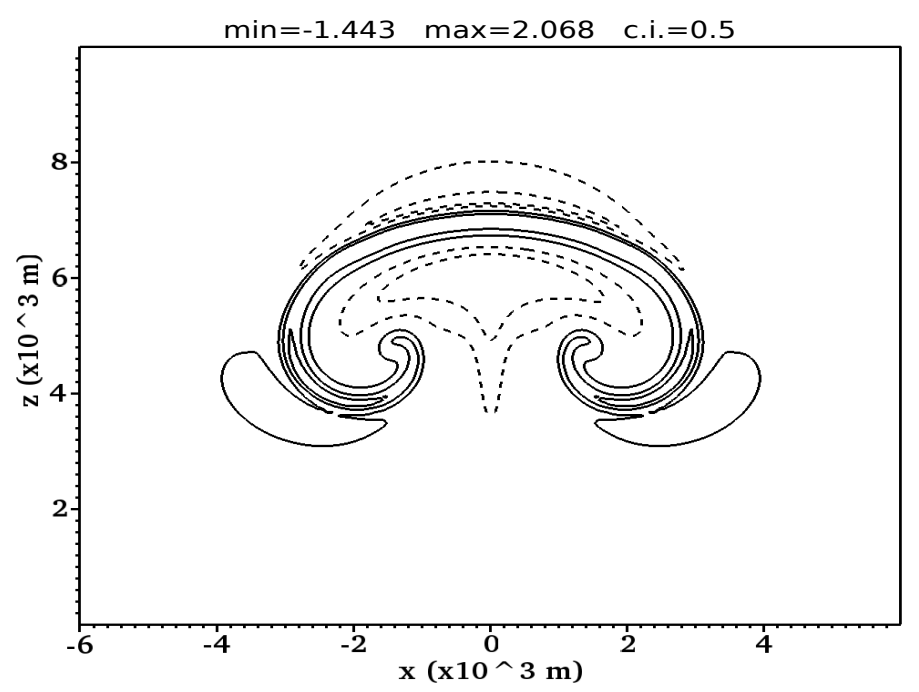

Figure 3: A contour plot of perturbations of the wet equivalent potential temperature at $t=1000 \mathrm{~s}$ for the moist bubble simulation at the higher resolution where the contour interval is $0.5 \mathrm{~K}$, the grid spacing is $66.67 \mathrm{~m}$ and the zero contour is omitted. 


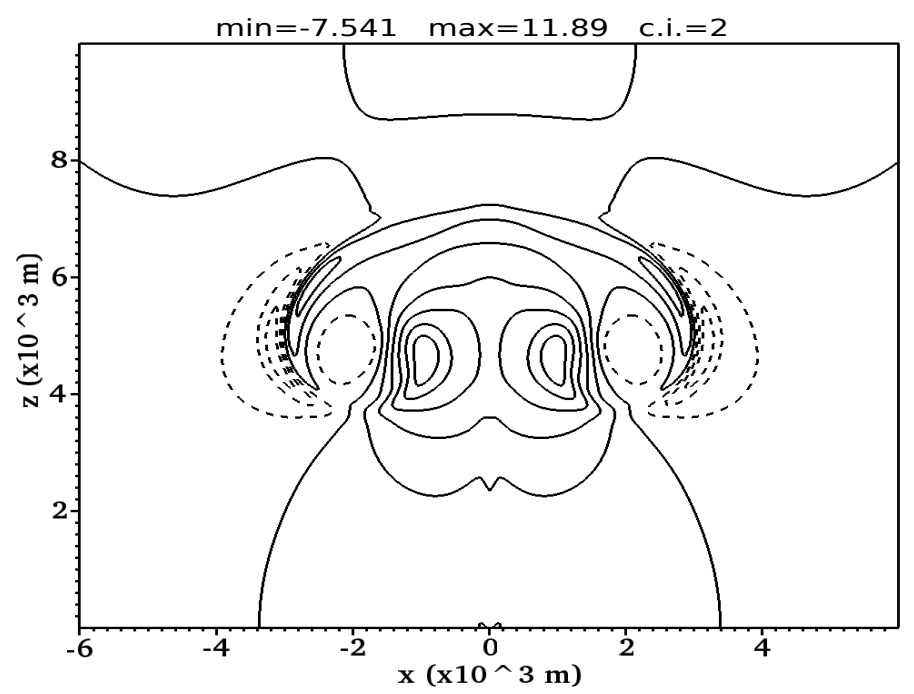

Figure 4: A contour plot of the vertical velocity at $t=1000 \mathrm{~s}$ for the moist bubble simulation at the higher resolution where the contour interval is $2 \mathrm{~ms}^{-1}$ and the grid spacing is $66.67 \mathrm{~m}$.

\section{Conclusions}

In the preceeding pages a pseudo-incompressible computational model which includes phase changes is developed. The addition of phase changes requires adjustments to the divergence constraint not seen in the more common anelastic model. Borrowing and adapting ideas from Almgren et al. (2008), these issues were tackled by allowing the background state to vary in time and then deriving a set of equations governing the evolution of the background variables.

The model was shown to perform well against a compressible model for the idealized bubble test-case presented in Bryan and Fritsch (2002). To compare the models contour plots of the vertical velocity and wet equivalent potential temperature are examined. The plots compare well overall but our 
bubble rises slight higher and results in some discrepancies in the form of the vertical velocity plot. Considering the fact that our models contain many differences in their numerics some variation is to be expected.

In deriving this model we have assumed that the pressure perturbations are small but unlike the anelastic equations no constraint was imposed on the size of the potential temperature or density variations. The assumption of small potential temperature and density variations is generally valid for moist atmospheric processes and as a result the moist pseudo-incompressible model may be expected to be superior to an anelastic model only in specific cases. For example, in the cases of deep convection and in the cases of baroclinic waves due to the retention of the baroclinic vorticity production term (see page 33 in Cotton et al. (2011)).

Regarding the microphysics, our usage of the initial pressure stratification in the caculation of the latent heat release is suitable for many atmospheric problems (including the test-case presented here) but may have to be modified when modelling larger-scale moist dynamics. It is possible to use a reconstructed full pressure in the source term calculation in sound-proof models as shown in Kurowski et al. (2013) and a similar approach to the one used in that paper may have to be implemented when using our model in problems where larger pressure perturbations are expected.

This work was motivated by the findings contained in Almgren et al. (2008) but there are some notable differences between the models. In Almgren's work an outflow top boundary is used whereas here we have given a method which is able to impose solid-wall boundary conditions. Another difference is that we have kept the coefficient in the divergence constraint 
in a physically meaningful form and did not have to result writing it as an integral. This is due to the fact that the $\gamma$ in the definition of potential temperature is constant and the coefficent will have to be written in a similar integral form when a non-constant $\gamma$ is required.

As far as the authors are aware this is the first implementation of a moist pseudo-incompressible model and we see it as being of great practicality for anyone modelling atmospheric processes for which larger perturbations are important. The reason being that the model is both more computationally efficient than a compressible model and holds greater validity than an anelastic model. Also, due to the fact that we do not use the Exner pressure in the momentum equation the model can be easily extened to a semi-implicit fully compressible model and by using the work presented in Klein and Pauluis (2011) thermodynamic consistency can also be ensured.

Acknowledgments. The authors would like to thank their colleagues Matthias Waidmann and Stephan Gerber for their work in developing the finite volume code, Ann Almgren for her support and feedback which greatly helped the development of this project, the Lawrence Livermore National Laboratory for the usage of their SAMRAI library, HYPRE library and VisIt visualisation program and especially the Berlin Mathematical School without whom this work wouldn't have been possible.

\section{Appendix A. Variable definitions}

Definition of variables and constants not defined in the text

$\rho$ - Total density 
$\mathbf{u}$ - Velocity of the air

$p$ - Total pressure

$q_{v}$ - Vapour mixing ratio

$q_{c}$ - Cloud water mixing ratio

$q=q_{v}+q_{c}-$ Total mixing ratio

$T$ - Temperature

$\theta$ - Potential temperature

$C$ - Condensation rate

$S$ - Latent heating rate

$g=9.81 \mathrm{~ms}^{-2}$ - Acceleration due to gravity

$p_{\text {ref }}=1.0 \times 10^{5} \mathrm{~Pa}$ - Reference pressure

$T_{r e f}=273.15 \mathrm{~K}$ - Reference temperature

$R=287 \mathrm{~J} \mathrm{~kg}^{-1} \mathrm{~K}^{-1}$ - Gas constant of dry air

$R_{v}=461 \mathrm{~J} \mathrm{~kg}^{-1} \mathrm{~K}^{-1}$ - Gas constant of water vapor

$c_{p}=1004.0 \mathrm{~J} \mathrm{~kg}^{-1} \mathrm{~K}^{-1}$ - Specific heat of dry air at constant pressure

$c_{p v}=1885 \mathrm{~J} \mathrm{~kg}^{-1} \mathrm{~K}^{-1}$ - Specific heat of water vapor at constant pressure

$c_{p c}=4186 \mathrm{~J} \mathrm{~kg}^{-1} \mathrm{~K}^{-1}$ - Specific heat of cloud water at constant pressure

$c_{v}=717 \mathrm{~J} \mathrm{~kg}^{-1} \mathrm{~K}^{-1}$ - Specific heat of dry air at constant volume

$L_{v 0}=2.5 \times 10^{6} \mathrm{~J} \mathrm{~kg}^{-1}$ - Latent heat of vaporisation reference value

$L_{v}=L_{v 0}-\left(c_{p c}-c_{p v}\right)\left(T-T_{r e f}\right)$ - Latent heat of vaporisation 


\section{Appendix B. Numerical details}

Appendix B.1. Explanation of the numerics notation

The notation used in this section is given as follows

$$
\begin{aligned}
& i \text { - Grid cell index in the horizontal direction } \\
& j \text { - Grid cell index in the vertical direction } \\
& x \text { - Horizontal position } \\
& z \text { - Vertical position } \\
& \Delta t \text { - Time step size } \\
& \Delta x \text { - Grid cell size in x-direction } \\
& \Delta z-\text { Grid cell size in x-direction } \\
& u \text { - Horizontal velocity } \\
& w-\text { Vertical velocity } \\
& n-\text { values at the end of previous time step } \\
& n+1 \text { - values at the end of current time step } \\
& p r e d-\text { values at the end of the predictor step }
\end{aligned}
$$

Appendix B.2. The predictor step

In this step the advective updates for $\rho, \rho \mathbf{u}, P, \rho q_{v}$ and $\rho q_{c}$ are calculated while ignoring the latent heat and condensation source terms. The time integration is preformed using a two step strong stability preserving RungeKutta scheme from Gottlieb et al. (2001). These steps can be written in general form as 
1. Step One

$$
\begin{aligned}
\phi_{i, j}^{*}= & \phi_{i, j}^{n}+\frac{\Delta t}{\Delta x}\left((\phi u)_{i+\frac{1}{2}, j}^{n}-(\phi u)_{i-\frac{1}{2}, j}^{n}\right) \\
& +\frac{\Delta t}{\Delta z}\left((\phi w)_{i, j+\frac{1}{2}}^{n}-(\phi w)_{i, j-\frac{1}{2}}^{n}\right)-\Delta t Q_{i, j}^{\phi}
\end{aligned}
$$

2. Step Two

$$
\begin{aligned}
\phi_{i, j}^{* *}= & \frac{1}{2}\left(\phi_{i, j}^{n}+\phi_{i, j}^{*}\right)+\frac{\Delta t}{2 \Delta x}\left((\phi v)_{i+\frac{1}{2}, j}^{*}-(\phi v)_{i-\frac{1}{2}, j}^{*}\right) \\
& +\frac{\Delta t}{2 \Delta z}\left((\phi w)_{i, j+\frac{1}{2}}^{*}-(\phi w)_{i, j-\frac{1}{2}}^{*}\right)-\frac{\Delta t}{2} Q_{i, j}^{\phi, *}
\end{aligned}
$$

where $\phi \in\left\{\rho, \rho u, \rho w, P, \rho q_{v}, \rho q_{c}\right\}$ and

$$
Q_{i, j}^{\phi}=\left\{\begin{array}{lr}
0 & \left(\phi \in\left\{\rho, P, \rho q_{v}, \rho q_{c}\right\}\right) \\
\left(p_{i+\frac{1}{2}, j}^{n}-p_{i-\frac{1}{2}, j}^{n}\right) & (\phi \in\{\rho u\}) \\
\left(p_{i, j+\frac{1}{2}}^{n}-p_{i, j-\frac{1}{2}}^{n}\right)+\rho_{i, j}^{n} g & (\phi \in\{\rho w\})
\end{array}\right.
$$

Note, $Q_{i, j}^{\phi, *}$ indicates that $\rho_{i, j}^{*}$ is to be used in the gravity term.

The fluxes in this step are calculated as follows, first the velocities on the cell faces are determined using

$$
\begin{aligned}
& u=\frac{1}{2}\left(u_{L}+u_{R}\right) \\
& w=\frac{1}{2}\left(w_{L}+w_{R}\right)
\end{aligned}
$$

where the $L$ and $R$ subscripts signify reconstructed values on the left and right of the interface. These values are reconstructed using a prescribed limiter. Finally, , fluxes are calculated in an upwind fashion. For example, to calculate $(\phi u)_{i+\frac{1}{2}, j}^{n}$ we let

$$
(\phi u)_{i+\frac{1}{2}, j}^{n}=\left((\phi u)_{i+\frac{1}{2}, j}^{n}\right)^{+}+\left((\phi u)_{i+\frac{1}{2}, j}^{n}\right)^{-}
$$


where

$$
\left((\phi u)_{i+\frac{1}{2}, j}^{n}\right)^{+}=\left(\phi_{i+\frac{1}{2}, j}^{n}\right)_{L} * \max (0, u)
$$

and

$$
\left((\phi u)_{i+\frac{1}{2}, j}^{n}\right)^{-}=\left(\phi_{i+\frac{1}{2}, j}^{n}\right)_{R} * \min (0, u)
$$

The remaining fluxes can be calculated using the same method.

It is important to note that we are using the pressure $p$ in the implementation of the momentum equation rather than the Exner pressure $\pi$ as is common in many "sound-proof" models.

Appendix B.3. Microphysics step

In this step we calculate the microphysical source terms and use them to update $\rho q_{v}, \rho q_{c}$ and $P$. Throughout this step we will be using the initial stratification of pressure in the calculation of temperature and the vapour saturation mixing ratio.

Following Grabowski and Smolarkiewicz (1990) the condensation rate in a single cell over a time-step can be calculated using

$$
C_{i, j}^{n}=\left[\frac{\left(q_{v}^{* *}-q_{v s}^{* *}\right)}{\left(1+\left(1+q_{v s}^{* *} / \epsilon\right)\left(\frac{q_{v s}^{* *} L_{v}^{n}}{c_{p}} \frac{L_{v 0}}{R_{v} T^{* * 2}}\right)\right) \Delta t}\right]_{i, j}
$$

where $q_{v s}$ is the vapour saturation mixing ratio, $T^{n}=\theta^{n}\left(p_{0}^{n} / p_{\text {ref }}\right)^{\left(R / c_{p}\right)}$ and $T^{* *}=\theta^{* *}\left(p_{0}^{n} / p_{r e f}\right)^{\left(R / c_{p}\right)}$. The vapour saturation mixing ratio can be calculated following Lipps and Helmer (1982) using

$$
q_{v s}=\frac{\epsilon e_{s}}{p_{0}-e_{s}}
$$


where the saturation vapour pressure is given by the

$$
e_{s}=e_{0} \exp \left(\frac{L_{v 0}}{R_{v}}\left(\frac{1}{T_{r e f}}-\frac{1}{T}\right)\right)
$$

with $e_{0}=611.0 \mathrm{~Pa}$.

Using $C_{i, j}$ we can now calculate the latent heat rate over one time-step as

$$
S_{i, j}^{n}=\left[\left(\frac{L_{v}}{c_{p} T}\right)-1 /\left(\epsilon+q_{v}\right)\right]_{i, j}^{n} P_{i, j}^{n} C_{i, j}^{n} .
$$

Finally, the microphysical updates are given as

$$
\begin{aligned}
& \left(\rho q_{v}\right)_{i, j}^{\text {pred }}=\left(\rho q_{v}\right)_{i, j}^{* *}-C_{i, j}^{n} \Delta t \\
& \left(\rho q_{c}\right)_{i, j}^{\text {pred }}=\left(\rho q_{v}\right)_{i, j}^{* *}+C_{i, j}^{n} \Delta t \\
& P_{i, j}^{\text {pred }}=P_{i, j}^{* *}+S_{i, j}^{n} \Delta t
\end{aligned}
$$

and now variables $\rho^{* *},(\rho u)^{* *}$ and $(\rho w)^{* *}$ will be written with a "pred" superscript since they also contain the predicted values.

\section{Appendix B.4. Background state update}

To calculate the background state at the new time level we must first calculate the rate of change of $p_{0}^{t o p}$ over the current time-step using (18). This is done as follows

$$
\left(\frac{\partial p_{0}^{t o p}}{\partial t}\right)^{n}=\frac{\sum_{j=j_{\min }}^{j_{\max }}\left(A V G(S) / P_{0}\right)_{j}^{n}}{\sum_{j=j_{\min }}^{j_{\max }}\left(1 / \gamma p_{0}\right)_{j}^{n}}
$$

where $A V G$ is the numerical approximation of the horizontal average and is given by $A V G(\cdot)=\frac{1}{\left(i_{\max }-i_{\min }\right)} \sum_{i=i_{\min }}^{i_{\max }}(\cdot)$. Note that any variables that have 
only a $j$ index do not depend on $x$. We then use this to calculate the background velocity on the interface from (19) using

$$
w_{0_{j+\frac{1}{2}}}^{n}=\Delta z\left[\sum_{k=j_{\text {min }}}^{j}\left(\left(A V G(S) / P_{0}\right)_{k}^{n}-\left(1 / \gamma p_{0}\right)_{k}^{n}\left(\frac{\partial p_{0}^{\text {top }}}{\partial t}\right)^{n}\right)\right]
$$

Finally, $P_{0}$ is calculated from (14) using

$$
\left(P_{0}\right)_{j}^{n+1}=\left(P_{0}\right)_{j}^{n}+\frac{\Delta t}{\Delta z}\left(\left(P_{0} w_{0}\right)_{j+1 / 2}^{n}-\left(P_{0} w_{0}\right)_{j-1 / 2}^{n}\right)+A V G(S)_{j}^{n} \Delta t .
$$

\section{Appendix B.5. First projection}

In this step the advective fluxes are corrected to satisfy the divergence constraint given by (13). From Klein (2009) we write a poisson equation which can be used to determine a pressure correction $\partial p$. The gradient of this pressure correction is used to correct the fluxes in the $P$ equation as follows

$$
\nabla \cdot(P \mathbf{u})_{i, j}^{n+1}=\nabla \cdot(P \mathbf{u})_{i, j}^{p r e d}-\frac{\Delta t}{2}\left(\nabla \cdot \frac{P^{\text {pred }}}{\rho^{\text {pred }}}\left(\nabla \partial p_{c}\right)\right)_{i, j} .
$$

Since the terms at $n+1$ satisfy the divergence constraint we re-write (B.17) as

$$
S_{i, j}^{n}-\left(\frac{P_{0}^{n+1}-P_{0}^{n}}{\Delta t}\right)_{j}=\nabla \cdot(P \mathbf{u})_{i, j}^{p r e d}-\frac{\Delta t}{2}\left(\nabla \cdot \frac{P^{\text {pred }}}{\rho^{\text {pred }}}\left(\nabla \partial p_{c}\right)\right)_{i, j}
$$

Now using the fact the $\left(\frac{P^{\text {pred }}-P^{n}}{\Delta t}\right)_{i, j}=S_{i, j}^{n}-\nabla \cdot(P \mathbf{u})_{i, j}^{\text {pred }}$ due to the predictor step we get

$$
\left(\frac{P^{\text {pred }}-P^{n}}{\Delta t}\right)_{i, j}-\left(\frac{P_{0}^{n+1}-P_{0}^{n}}{\Delta t}\right)_{j}=-\frac{\Delta t}{2}\left(\nabla \cdot \frac{P^{\text {pred }}}{\rho^{\text {pred }}}\left(\nabla \partial p_{c}\right)\right)_{i, j} .
$$


Since the old time level values have already been corrected we know that $P_{i, j}^{n}=P_{0}^{n}$ and we get

$$
\left(\frac{\Delta t}{2} \nabla \cdot \frac{P^{\text {pred }}}{\rho^{\text {pred }}}\left(\nabla \partial p_{c}\right)\right)_{i, j}=-\left(\frac{P_{i, j}^{\text {pred }}-P_{0_{j}}^{n+1}}{\Delta t}\right) .
$$

Solving this equation using using subroutines from the HYPRE package (see Falgout and Yang (2002)) to solve the Poisson equation, we write the flux update to the $P$ equation as

$$
(\partial(P \mathbf{u}) \cdot \mathbf{n})_{i, j}=\left(\frac{\Delta t}{2} \frac{P^{p r e d}}{\rho^{p r e d}}\left(\nabla \partial p_{c}\right) \cdot \mathbf{n}\right)_{i, j}
$$

and then the advected variables are updated as follows

$$
\begin{aligned}
\phi_{i, j}^{n+1}= & \phi_{i, j}^{\text {pred }}+\frac{\Delta t}{\Delta x}\left(\left(\partial(P u) \frac{\phi^{\text {pred }}}{P^{\text {pred }}}\right)_{i+\frac{1}{2}, j}-\left(\partial(P u) \frac{\phi^{\text {pred }}}{P^{\text {pred }}}\right)_{i-\frac{1}{2}, j}\right) \\
& +\frac{\Delta t}{\Delta z}\left(\left(\partial(P w) \frac{\phi^{\text {pred }}}{P^{\text {pred }}}\right)_{i, j+\frac{1}{2}}-\left(\partial(P w) \frac{\phi^{\text {pred }}}{P^{\text {pred }}}\right)_{i, j-\frac{1}{2}}\right)^{2}
\end{aligned}
$$

for $\phi \neq \rho u, \rho w$. The only difference for $\phi=\rho u, \rho w$ is that the updated values will be labeled $\phi^{\text {firstPro }}$ instead of $\phi^{n+1}$ of since they are not yet the fully corrected values.

\section{Appendix B.6. Second projection}

Here the cell-centered momenta are corrected by an increment of the nodal pressure. This pressure increment is determined again through a Poisson equation that is derived from the divergence constraint divergence constraint given by (13). Also using Klein (2009) we can write a poisson equation on 
the nodes for the pressure correction

$$
\begin{aligned}
\left(\frac{\Delta t}{2} \nabla \cdot\left(\frac{P}{\rho}\right)^{n+1} \nabla(\partial p)\right)_{i+\frac{1}{2}, j+\frac{1}{2}}= & \nabla(P \mathbf{u})_{i+\frac{1}{2}, j+\frac{1}{2}}^{\text {firstPro }} \\
& -\left(S_{i+\frac{1}{2}, j+\frac{1}{2}}^{n}-\frac{P_{0_{j+\frac{1}{2}}^{n+1}}^{n}-P_{0_{j+\frac{1}{2}}^{n}}^{n}}{\Delta t}\right)
\end{aligned}
$$

where the values on the right hand side are interpolated from the cell centered values. After solving for $\partial p$ using the poisson solver the velocity is updated using

$$
\begin{aligned}
(\rho u)_{i, j}^{n+1}= & (\rho u)_{i, j}^{\text {firstPro }}-\frac{\Delta t}{2 \Delta x}\left(\left(\partial p_{i+\frac{1}{2}, j+\frac{1}{2}}+\partial p_{i+\frac{1}{2}, j-\frac{1}{2}}\right)\right. \\
& \left.-\left(\partial p_{i-\frac{1}{2}, j+\frac{1}{2}}+\partial p_{i-\frac{1}{2}, j-\frac{1}{2}}\right)\right)
\end{aligned}
$$

and

$$
\begin{aligned}
(\rho w)_{i, j}^{n+1}= & (\rho w)_{i, j}^{f i r s t P r o}-\frac{\Delta t}{2 \Delta z}\left(\left(\partial p_{i+\frac{1}{2}, j+\frac{1}{2}}+\partial p_{i-\frac{1}{2}, j+\frac{1}{2}}\right)\right. \\
& \left.-\left(\partial p_{i+\frac{1}{2}, j-\frac{1}{2}}+\partial p_{i-\frac{1}{2}, j-\frac{1}{2}}\right)\right) .
\end{aligned}
$$

Now all our variables are second order accurate at the new time level and they also satisfy the divergence constraint.

Almgren, A. S., 2000. A new look at the pseudo-incompressible solution to lamb's problem of hydrostatic adjustment. Journal of the Atmospheric Sciences 57, 995-998.

Almgren, A. S., Bell, J. B., C. A. Rendleman, ., Zingale, M., 2008. Low mach number modeling of type ia x-ray supernovae iii. reactions. The Astrophysical Journal 684, 449-470. 
Boussinesq, J. V., 1903. Théorie des Analytique de la Chaleur. Vol. 2. Gauthier-Villars.

Bryan, G. H., Fritsch, J. M., Dec. 2002. A benchmark simulation for moist nonhydrostatic numerical models. Mon. Wea. Rev. 130 (12), 2917-2928.

Clark, T. L., Jun. 1977. A small-scale dynamic model using a terrainfollowing coordinate transformation. Journal of Computational Physics 24, 186-215.

Cotton, W. R., Bryan, G., van der Heever, S. C., 2011. Storm and Cloud Dynamics. Academic Press.

Durran, D., 1989. Improving the anelastic approximation. Journal of the Atmospheric Sciences 49, 1453-1461.

Emanuel, K. A., 1994. Atmospheric Convection. Oxford University Press.

Falgout, R., Yang, U., 2002. hypre: A library of high performance preconditioners. In: Sloot, P., Hoekstra, A., Tan, C., Dongarra, J. (Eds.), Computational Science - ICCS 2002. Vol. 2331 of Lecture Notes in Computer Science. Springer Berlin Heidelberg, pp. 632-641.

Gottlieb, S., Shu, C.-W., Tadmor, E., 2001. Strong stability-preserving highorder time discretization methods. SIAM Review 43, 89-112.

Grabowski, W. W., Smolarkiewicz, P. K., 1990. Monotone finite-difference approximations to the advection-condenstaion problem. Monthly Weather Review 118, 2082-2097. 
Grabowski, W. W., Smolarkiewicz, P. K., 2002. A multiscale anelastic model for meteorological research. Monthly Weather Review 130, 939-956.

Klein, R., 2009. Asymptotics, structure, and integration of sound-proof atmospheric flow equations. Theoretical and Computational Fluid Dynamics 23, 161-195.

Klein, R., Pauluis, O., 2011. Thermodynamic consistency of a pseudoincompressible approximation for general equations of state. Journal of the Atmospheric Sciences 69, 961-968.

Kurowski, M. J., Grabowski, W. W., Smolarkiewicz, P. K., 2013. Towards multiscale simulation of moist flows with soundproof equations. Journal of the Atmospheric Sciences.

Lipps, F. B., Helmer, R. S., 1982. A scale analysis of deep moist convection and some related numerical calculations. Journal of Atmospheric Sciences 29, 2192-2210.

Ogura, Y., Phillips, N. A., 1962. Scale analysis of deep and shallow convection in the atmosphere. Journal of Atmospheric Sciences 19, 173-179.

Pauluis, O., 2008. Thermodynamic consistency of the anelastic approximation for a moist atmosphere. Journal of the Atmospheric Sciences 65, 2719-2729.

Straka, J. M., Wilhelmson, R. B., Wicker, L. J., Anderson, J. R., Droegemeier, K. K., Jul. 1993. Numerical solutions of a non-linear density current: A benchmark solution and comparisons. International Journal for Numerical Methods in Fluids 17, 1-22. 